$\underline{\text { Article }}$

\title{
Xanthones and Other Constituents of Vismia parviflora
}

\author{
Tanus Jorge Nagem ${ }^{a}$, and Fernando Faustino de Oliveira ${ }^{b}$ \\ ${ }^{a}$ Departamento de Química, Universidade Federal de Ouro Preto, Campus Morro do \\ Cruzeiro, 35400-000 Ouro Preto - MG, Brazil \\ ${ }^{b}$ Departamento de Química-ICEx, Universidade Federal de Minas Gerais, Av. Antônio \\ Carlos, 6627 Pampulha, 31270-010 Belo Horizonte - MG, Brazil
}

Received: August 28, 1996

\begin{abstract}
Vismia parviflora, planta da família Guttiferae, é uma espécie da tribo Vismeae encontrada na região de Ouro Preto MG. Dos extratos benzênico e etanólico dos galhos e etanólico dos frutos de um especimen desta planta, cromatografados em sílica gel, foram isolados sitosterol, ácido betulínico, lupeol, friedelina, $\beta$-friedelinol, ácido chiquímico, ácido 3,4-diidroxibenzóico, quercetina e as antraquinonas madagascina, ácido crisofânico, vismiaquinona-A e vismiaquinona-C, além das xantonas 1,7-diidroxixantona e 1,5-diidroxi-8-metoxixantona. Estas substâncias tiveram suas estruturas elucidadas com base nos seus dados espectrométricos de IV, UV, EM e RMN de ${ }^{1} \mathrm{H}$ e ${ }^{13} \mathrm{C}$ uni-(1D) e bidimensional-(2D).
\end{abstract}

The stems and fruits of Vismia parviflora have been shown to contain sitosterol, betulinic acid, lupeol, friedelin, $\beta$-friedelinol, shikimic acid, 3,4-dihydroxybenzoic acid, quercetin, 1,7-dihydroxyxanthone, 1,5-dihydroxy-8-methoxyxanthone, madagascine, chrysophanic acid, vismiaquinone-A and its isomer vismiaquinone- $\mathrm{C}$. The structures of these compounds have been elucidated by using spectroscopic data as MS, UV, IR, one- and two-dimensional NMR.

Keywords: Vismia parviflora, Guttiferae, xanthone, anthraquinone, quercetin, terpenoids

\section{Introduction}

According to Engler ${ }^{1}$, Vismia parviflora belongs to the Guttiferae family, subfamily Hyperiocoideae, tribe Vismieae. The Vismia genus consists of small trees inhabiting the tropical and subtropical regions of South and Central America, where they are used in folk medicine as strong purgative, whereas their barks are considered to be tonic and febrifugal ${ }^{2-3}$.

Although the chemistry of the Guttiferae family has been widely studied $^{4}$, no more than fifteen species of Vismia have been investigated among the fifty known species. Several species of this genus have been shown to contain xanthones and anthranoides ${ }^{5-10}$. In the present study we have identified the chemical constituents from stems and fruits of Vismia parviflora, which have not been reported previously.

\section{Results and Discussion}

Fractionation of the ethanol extracts of stems and fruits on silica gel, followed by gel filtration and recrystalization, afforded the isolation the compounds 1-6. The UV spectra of $\mathbf{1}$ and $\mathbf{2}$ exhibited absorption bands characteristic of xanthones ${ }^{11}$. The bathochromic shift observed upon addition of $\mathrm{AlCl}_{3}$ indicated the presence of a chelated hydroxyl group at $\mathrm{C}-1$ or $\mathrm{C}-8$ for the both compounds $\mathbf{1}$ and $\mathbf{2}$. The ${ }^{1} \mathrm{H}-\mathrm{NMR}$ spectra of both $\mathbf{1}$ and $\mathbf{2}$ showed, absorptions at: $\delta 7.68(\mathrm{t}, \mathrm{J}=8.1 \mathrm{~Hz} \mathrm{H}-3), 6.75$ and 6.97(dd, $\mathrm{J}=8.1$ and 1,0 $\mathrm{Hz} \mathrm{H}-2$ and $\mathrm{H}-4)$ for 1 and $\delta 7.63(\mathrm{t}, \mathrm{J}=7.30 \mathrm{~Hz} \mathrm{H}-3), 6.72$ and $6.94(\mathrm{dd}, \mathrm{J}=7.3$ and $1.3 \mathrm{~Hz} \mathrm{H}-2$ and $\mathrm{H}-4)$ for 2 , indicative for both compounds the same three hydrogens vicinal pattern for one of the aromatic ring. In addition, the ${ }^{1} \mathrm{H}-\mathrm{NMR}$ of compound 1 showed three ortho- and metacoupled hydrogens at $\delta 7.50(\mathrm{~d}, \mathrm{~J}=8.8 \mathrm{~Hz} \mathrm{H}-5), 7.40$ (dd, $\mathrm{J}=8.8$ and $2.7 \mathrm{~Hz} \mathrm{H}-6), 7.57$ (d, $\mathrm{J}=2.7 \mathrm{~Hz} \mathrm{H}-8)$. The $\mathrm{OH}$ on $\mathrm{C}-7$ was proposed by the absence of the bathochromic shift of the UV maxima in the presence of sodium acetate, and confirmed by methylation of $\mathbf{1}$ with an ether solution of diazomethane. The ${ }^{13} \mathrm{C}-\mathrm{NMR}$ spectra was compatible with 1,7-dihydroxyxanthone $\mathbf{1}$. The mass spectrum showed 
Table 1. ${ }^{1} \mathrm{H}-\mathrm{NMR}(200 \mathrm{MHz})$ and ${ }^{13} \mathrm{C}-\mathrm{NMR}(50 \mathrm{MHz})$ spectral data for the compounds 1-3 compared with literature 1a-3a.

\begin{tabular}{|c|c|c|c|c|c|c|c|c|c|c|c|c|}
\hline \multirow{3}{*}{$\mathrm{C}$} & \multicolumn{4}{|c|}{ Compound 1} & \multicolumn{4}{|c|}{ Compound 2} & \multicolumn{4}{|c|}{ Compound 3} \\
\hline & \multicolumn{2}{|c|}{1 (acetone- $\left.\mathrm{d}_{6}\right)$} & \multicolumn{2}{|c|}{$\begin{array}{c}\text { 1a lit. }[15]^{\mathrm{Q}} \\
\left(\mathrm{CDCl}_{3}\right)\end{array}$} & \multicolumn{2}{|c|}{2 (acetone- $\left.\mathrm{d}_{6}\right)$} & \multicolumn{2}{|c|}{$\begin{array}{c}\text { 2a lit. }[14] \# \\
\left(\mathrm{CDCl}_{3} / \mathrm{CD}_{3} \mathrm{OD}\right. \\
9: 1)\end{array}$} & \multicolumn{2}{|c|}{$3\left(\mathrm{CDCl}_{3}\right)$} & \multicolumn{2}{|c|}{$\begin{array}{c}\text { 3a lit. }[16]^{!} \\
\left(\mathrm{CDCl}_{3}\right)\end{array}$} \\
\hline & $\delta^{13} \mathrm{C}$ & $\delta^{1} \mathrm{H}$ & $\begin{array}{c}\delta \\
{ }^{13} \mathrm{C}^{*}\end{array}$ & $\delta{ }^{1} \mathrm{H}$ & $\delta^{13} \mathrm{C}$ & $\delta^{1} \mathrm{H}$ & $\delta^{13} \mathrm{C}^{*}$ & $\delta^{1} \mathrm{H}$ & $\delta^{13} \mathrm{C}$ & $\delta{ }^{1} \mathrm{H}$ & $\delta^{13} \mathrm{C}^{*}$ & $\delta^{1} \mathrm{H}$ \\
\hline 1 & 163.0 & --- & & --- & 161.9 & --- & & --- & 162.4 & --- & & --- \\
\hline 2 & 110.4 & $\begin{array}{c}6.75 \\
\operatorname{dd}(1.0 \\
8.1)^{\S}\end{array}$ & & $\begin{array}{c}6.77 \\
\operatorname{dd}(0.5 \\
8.4)\end{array}$ & 111.1 & $\begin{array}{c}6.72 \\
\operatorname{dd}(1.3 \\
7.3)\end{array}$ & & $\begin{array}{c}6.76 \\
\operatorname{dd}(1.5 ; 8.0)\end{array}$ & 124.5 & $7.06 \mathrm{sl}$ & & 7.08 \\
\hline 3 & 138.0 & $7.68 \mathrm{t}(8.1)$ & & $7.59 \mathrm{t}(8.8)$ & 136.1 & $7.63 \mathrm{t}(7.3)$ & & $\begin{array}{c}7.56 \\
\operatorname{dd}(1.5 ; 8.0)\end{array}$ & 136.9 & --- & & --- \\
\hline 4 & 107.0 & $\begin{array}{c}6.97 \\
\operatorname{dd}(1.0 \\
8.1)\end{array}$ & & $\begin{array}{c}6.95 \\
\mathrm{dd}(0.5 \\
8.4)\end{array}$ & 106.1 & $\begin{array}{c}6.94 \\
\operatorname{dd}(1.3 \\
7.3)\end{array}$ & & $\begin{array}{c}6.96 \\
\operatorname{dd}(1.5 ; 8.5)\end{array}$ & 124.4 & $7.61 \mathrm{sl}$ & & 7.61 \\
\hline $4 a$ & 157.0 & --- & & --- & 153.1 & --- & & --- & 133.1 & --- & & --- \\
\hline $4 b$ & 131.0 & --- & & --- & 145.7 & --- & & --- & 133.2 & --- & & --- \\
\hline 5 & 119.5 & $7.50 \mathrm{~d}(8.8)$ & & $7.41 \mathrm{~d}(9.3)$ & 138.5 & --- & & --- & 118.1 & $7.78 \mathrm{~d}(8.0)$ & & 7.80 \\
\hline 6 & 126.0 & $\begin{array}{c}7.40 \\
\operatorname{dd}(2.7 \\
8.8)\end{array}$ & & $\begin{array}{c}7.33 \\
\text { dd(2.9; } \\
9.3)\end{array}$ & 121.1 & $7.32 \mathrm{~d}(7.3)$ & & $7.26 \mathrm{~d}(9.0)$ & 121.3 & $7.64 \mathrm{t}(8.0)$ & & 7.65 \\
\hline 7 & 154.7 & --- & & --- & 105.1 & $6.85 \mathrm{~d}(7.3)$ & & $6.66 \mathrm{~d}(9.0)$ & 119.9 & $7.25 \mathrm{~d}(8.0)$ & & 7.27 \\
\hline 8 & 108.0 & $7.57 \mathrm{~d}(2.7)$ & & $7.63 \mathrm{~d}(2.9)$ & 154.8 & --- & & --- & 162.6 & --- & & --- \\
\hline $8 \mathrm{a}$ & 121.0 & --- & & --- & 109.0 & --- & & --- & 108.5 & --- & & --- \\
\hline $8 b$ & 109.0 & --- & & --- & 110.7 & --- & & --- & 107.3 & --- & & --- \\
\hline 9 & 181.5 & --- & & --- & 182.5 & --- & & --- & 191.8 & --- & & --- \\
\hline 10 & --- & --- & --- & --- & --- & --- & --- & --- & 181.7 & --- & & --- \\
\hline $\mathrm{OH}$ & --- & $\begin{array}{c}12.57 \\
9.20\end{array}$ & --- & $\begin{array}{c}12.62 \\
5.01\end{array}$ & --- & $\begin{array}{c}13.17 \\
8.70\end{array}$ & --- & 12.97 & --- & $\begin{array}{l}12.10 \\
11.98\end{array}$ & & $*$ \\
\hline $\mathrm{OMe}$ & --- & --- & --- & --- & 56.3 & $3.89 \mathrm{~s}$ & & $3.85 \mathrm{~s}$ & --- & --- & & --- \\
\hline $\mathrm{Me}$ & --- & --- & --- & --- & --- & --- & --- & --- & 22.2 & $2.44 \mathrm{~s}$ & & $*$ \\
\hline
\end{tabular}

§-Coupling constants $\left(\mathrm{J}\right.$ in Hz) are given in parentheses. $\Theta-{ }^{1} \mathrm{H}-\mathrm{NMR}(200 \mathrm{MHz}) . \#-{ }^{1} \mathrm{H}-\mathrm{NMR}(60 \mathrm{MHz}) . !$ - ${ }^{1} \mathrm{H}-\mathrm{NMR}(300 \mathrm{MHz}) . *$ - No value is provided.

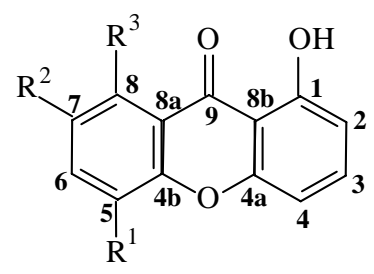

$1 \quad \mathrm{R}^{1}=\mathrm{R}^{3}=\mathrm{H} ; \mathrm{R}^{2}=\mathrm{OH}$

$2 \quad \mathrm{R}^{1}=\mathrm{OH} ; \mathrm{R}^{2}=\mathrm{H} ; \mathrm{R}^{3}=\mathrm{OMe}$<smiles>[R]c1cc2c(c(O)c1[R])C(=O)c1cc(C)cc(O)c1C2=O</smiles>

$3 \mathrm{R}^{1}=\mathrm{R}^{2}=\mathrm{H}$

$4 \mathrm{R}^{1}=\mathrm{H} ; \mathrm{R}^{2}=\mathrm{O}_{2^{\prime}}^{\mathrm{I}^{\prime}}$

$5 \mathrm{R}^{1}=\overbrace{2^{\prime}}^{1^{\prime}} \int_{5^{\prime}}^{4^{\prime}} ; \mathrm{R}^{2}=\mathrm{OMe}$

$6 \mathrm{R}^{1}=\overbrace{2^{\prime}}^{1^{\prime}} \int_{5^{\prime}}^{4^{\prime}} ; \mathrm{R}^{2}=\mathrm{OMe}$

Figure 1. Xanthones and anthraquinones isolated from Vismia parviflora. 
Table 2. ${ }^{1} \mathrm{H}-\mathrm{NMR}(200 \mathrm{MHz})$ and ${ }^{13} \mathrm{C}$ NMR $(50 \mathrm{MHz})$ spectral data for the compounds 4-6 compared with literature 4a-6a.

\begin{tabular}{|c|c|c|c|c|c|c|c|c|c|c|c|c|}
\hline \multicolumn{5}{|c|}{ Compound 4} & \multicolumn{4}{|c|}{ Compound 5} & \multicolumn{4}{|c|}{ Compound 6} \\
\hline \multirow[t]{2}{*}{$\mathrm{C}$} & \multicolumn{2}{|c|}{$4\left(\mathrm{CDCl}_{3}\right)$} & \multicolumn{2}{|c|}{ 4a lit. $[7]^{\#}\left(\mathrm{CDCl}_{3}\right)$} & \multicolumn{2}{|c|}{$5\left(\mathrm{CDCl}_{3}\right)$} & \multicolumn{2}{|c|}{$\begin{array}{c}\text { 5a lit. }[10]^{!} \\
\left(\mathrm{CDCl}_{3}\right)\end{array}$} & \multicolumn{2}{|c|}{$6\left(\mathrm{CDCl}_{3}\right)$} & \multicolumn{2}{|c|}{ 6a lit. $[8]^{!}\left(\mathrm{CDCl}_{3}\right)$} \\
\hline & $\delta^{13} \mathrm{C}$ & $\delta^{1} \mathrm{H}$ & $\delta^{13} \mathrm{C}^{*}$ & $\delta^{1} \mathrm{H}$ & $\delta^{13} \mathrm{C}$ & $\delta^{1} \mathrm{H}$ & $\delta^{13} \mathrm{C}^{*}$ & $\delta{ }^{1} \mathrm{H}$ & $\delta^{13} \mathrm{C}$ & $\delta^{1} \mathrm{H}$ & $\delta^{13} \mathrm{C}$ & $\delta^{1} \mathrm{H}$ \\
\hline 1 & 164.9 & - & & - & 163.9 & - & & - & 162.8 & - & 162.6 & - \\
\hline 2 & 124.3 & $6.56 \mathrm{~d}(2.0)$ & & $6.50 \mathrm{~d}(2.0)$ & 124.8 & $7.04 \mathrm{~d}(1.6)$ & & $7.02 \mathrm{~s}$ & 124.3 & $6.93 \mathrm{sl}$ & 124.2 & $7.03 \mathrm{~s}$ \\
\hline 3 & 139.7 & - & & - & 133.6 & - & & - & 148.3 & - & 148.1 & - \\
\hline 4 & 121.1 & $7.23 \mathrm{~d}(2.0)$ & & $7.13 \mathrm{~d}(2.0)$ & 121.0 & $7.58 \mathrm{~d}(1.6)$ & & $7.55 \mathrm{~s}$ & 121.0 & $7.24 \mathrm{sl}$ & 121.0 & $7.34 \mathrm{~s}$ \\
\hline $4 a$ & 133.1 & - & & - & 133.6 & - & & - & 131.9 & - & 133.0 & - \\
\hline $4 b$ & 135.0 & - & & - & 133.4 & - & & - & 133.0 & - & $*$ & - \\
\hline 5 & 107.4 & $7.50 \mathrm{~d}(1.8)$ & & $7.43 \mathrm{~d}(2.0)$ & 103.7 & $7.34 \mathrm{~s}$ & & $7.35 \mathrm{~s}$ & 110.3 & $7.46 \mathrm{~s}$ & 103.1 & $7.56 \mathrm{~s}$ \\
\hline 6 & 165.7 & - & & - & 162.1 & - & & - & 161.9 & - & 161.8 & - \\
\hline 7 & 108.6 & $6.91 \mathrm{~d}(1.8)$ & & $6.95 \mathrm{~d}(2.0)$ & 124.6 & - & & - & 114.0 & - & 115.7 & - \\
\hline 8 & 162.3 & - & & - & 162.8 & - & & - & 162.3 & - & 162.2 & - \\
\hline $8 \mathrm{a}$ & 108.0 & - & & - & 111.1 & - & & - & 110.4 & - & 110.3 & - \\
\hline $8 b$ & 110.0 & - & & - & 114.1 & - & & - & 115.7 & - & 113.5 & - \\
\hline 9 & 190.5 & - & & - & 191.7 & - & & - & 191.2 & - & 190.9 & - \\
\hline 10 & 182.0 & - & & - & 182.7 & - & & - & 181.7 & - & 182.4 & - \\
\hline $1^{\prime}$ & 65.6 & $\begin{array}{c}4.54 \\
\mathrm{~d}(6.4)^{\S}\end{array}$ & & $4.58 \mathrm{~d}(7.0)$ & 22,1 & $3.40 \mathrm{~d}(7.0)$ & & $3.40 \mathrm{~d}(7.0)$ & 132.0 & $\begin{array}{c}6.50 \\
d(16.2)\end{array}$ & 131.8 & $\begin{array}{c}6.60 \\
d(16.0)\end{array}$ \\
\hline $2^{\prime}$ & 118.1 & $\begin{array}{c}5.40 \\
\operatorname{dd}(6.7)\end{array}$ & & $5.40 \mathrm{t}(7.0)$ & 121.5 & $5.16 \mathrm{t}(7.0)$ & & $5.18 \mathrm{t}(7.0)$ & 146.6 & $\begin{array}{c}6.85 \\
\operatorname{dd}(7.0 \\
16.2)\end{array}$ & 146.5 & $\begin{array}{c}6.95 \\
\mathrm{dd}(6.5 \\
16.0)\end{array}$ \\
\hline $3^{\prime}$ & 143.5 & - & & - & 126.3 & - & & - & 33.4 & $2.40 \mathrm{~m}$ & 33.4 & $2.48 \mathrm{~m}$ \\
\hline $4^{\prime}$ & 25.8 & $1.71 \mathrm{~s}$ & & $1.75 \mathrm{~s}$ & 22.6 & $1.66 \mathrm{~s}$ & & $1.68 \mathrm{~s}$ & 22.7 & $1.04 \mathrm{~d}(7.0)$ & 22.5 & $1.14 \mathrm{~d}(6.5)$ \\
\hline 5 & 18.3 & $1.75 \mathrm{~s}$ & & $1.75 \mathrm{~s}$ & 22.5 & $1.78 \mathrm{~s}$ & & $1.80 \mathrm{~s}$ & 22.4 & $1.05 \mathrm{~d}(7.0)$ & 22.5 & $1.14 \mathrm{~d}(6.5)$ \\
\hline $\mathrm{OH}$ & - & $\begin{array}{l}12.26 \\
12.10\end{array}$ & - & $\begin{array}{l}12.03 \\
11.90\end{array}$ & - & $\begin{array}{l}12.39 \\
12.12\end{array}$ & - & $\begin{array}{l}12.40 \\
12.12\end{array}$ & - & $\begin{array}{c}12.80 \\
11.96\end{array}$ & - & $\begin{array}{l}12.84 \\
12.02\end{array}$ \\
\hline $\mathrm{Me}-3$ & 22.1 & $2.34 \mathrm{~s}$ & & $2.38 \mathrm{~s}$ & 20.5 & $2.42 \mathrm{~s}$ & & $2.44 \mathrm{~s}$ & 22.1 & $2.34 \mathrm{~s}$ & 22.1 & $2.42 \mathrm{~s}$ \\
\hline $\mathrm{OMe}$ & - & - & - & - & 56.3 & $4.01 \mathrm{~s}$ & & $4.00 \mathrm{~s}$ & 56.2 & $3.93 \mathrm{~s}$ & 56.1 & $4.02 \mathrm{~s}$ \\
\hline
\end{tabular}

$\S$ - Coupling constants ( $\mathrm{J}$ in Hz) are given in parentheses. \# - ${ }^{1} \mathrm{H}-\mathrm{NMR}(60 \mathrm{MHz})$. ! - ${ }^{1} \mathrm{H}-\mathrm{NMR}(100 \mathrm{MHz}) . *$ - No value is provided.

peak at $\mathrm{m} / \mathrm{z} 228\left([\mathrm{M}]^{+\bullet}\right)$, which is in accordance with the molecular formula $\mathrm{C}_{13} \mathrm{H}_{8} \mathrm{O}_{4}$. For the compound 2 the ${ }^{1} \mathrm{H}$ NMR showed two ortho coupled hydrogens at $\delta 6.85$ and 7.32 (d, J = 7.3 Hz, H-7 and H-6 respectivelly), besides the singlet at $\delta 3.89\left(3 \mathrm{H}, \mathrm{s}, \mathrm{OCH}_{3}\right)$. The ${ }^{13} \mathrm{C}-\mathrm{NMR}$ was compatible with 1,5-dihydroxy-8-methoxyxanthone. The mass spectrum showed peak at $\mathrm{m} / \mathrm{z} 258\left([\mathrm{M}]^{+\bullet}, 96 \%\right)$ in accordance with molecular formula $\mathrm{C}_{14} \mathrm{H}_{10} \mathrm{O}_{5}$, and the base peak at $\mathrm{m} / \mathrm{z} 240\left([\mathrm{M}-18]^{+\bullet}, 100 \%\right)$. The loss of water from the $[\mathrm{M}]^{+\bullet}$ is due to the operation of an ortho effect caused by the methoxy substituent at C- $8^{12}$, what is in agreement with the proposed structure. (Table 1).

Compounds 3-6, appeared to be 1,8-dihydroxyanthraquinone derivatives based on their UV-Vis and the IR absorptions bands characteristic of anthraquinones ${ }^{13}$. The ${ }^{1} \mathrm{H}-\mathrm{NMR}$ spectra of these compounds showed the same pattern of substitution for one of the aromatic ring, all of them showing two meta-coupling hydrogens, between $\delta$ 7.23 - $7.61(\mathrm{H}-4)$ and 6.59-7.06 (H-2), besides the methyl group at $\mathrm{C}-3$ in $\delta 2.4$ and a chelated hydroxy group at C-1 for all of them. The ${ }^{1} \mathrm{H}$-NMR spectra of compounds 4 and 5 showed signals compatible with a $\gamma, \gamma^{6}$-dimethylallyl side chain, an O-dimethylallyl at C-6 for 4, whereas 5 has a dimethylallyl at C-7. In addition compound $\mathbf{5}$ showed a methoxy group at 4.01 and an aromatic hydrogen at $\delta 7.34$ (s, H-5). For compound 6 the ${ }^{1} \mathrm{H}-\mathrm{NMR}$ spectra showed signals in accordance with the presence of a $\Delta^{1}$ - isopentenyl 
side chain at $\mathrm{C}-7$, a methoxy group at $\delta 3.93$ and an aromatic hydrogen at $\delta 7.46(\mathrm{~s}, \mathrm{H}-5)$. (Table 2).

\section{Experimental}

Plant material, A specimen of Vismia was collected in Três Moinhos district, Ouro Preto city, State of Minas Gerais, Brazil, in March 1986. The specimen was identified by Prof. José Badini, botanic garden of the Universidade Federal de Ouro Preto - Brazil.

Extraction and isolation of the constituents from stems: Air-dried, powdered stems (1,400 g) were extracted with $\mathrm{C}_{6} \mathrm{H}_{6}$ followed by EtOH. Removal of the solvents gave 9.4 $\mathrm{g}$ and $22.0 \mathrm{~g}$ as residues respectively. The benzene extract was chromatographed on silica gel (200.0 g), elution was performed with $\mathrm{C}_{6} \mathrm{H}_{6}$, EtOAc and EtOH. Several frs. were collected and sepd. into ten groups $\left(\mathrm{A}_{1}-\mathrm{A}_{10}\right)$, by TLC. $\mathrm{A}_{1}$ $(0.36 \mathrm{~g})$ was purified by rechromatography and recrystalization from acetone yielding friedelin $(10.0 \mathrm{mg})$ and $\beta$ friedelinol $(7.0 \mathrm{mg}) . \mathrm{A}_{7}(0.20 \mathrm{~g})$ was rechromatographed on silica gel $(10.0 \mathrm{~g})$, using $\mathrm{C}_{6} \mathrm{H}_{6}, \mathrm{CHCl}_{3}$ and EtOAc as eluents, giving sitosterol ( $24.4 \mathrm{mg}$,), 1,7-dihydroxyxantone (1, $1.5 \mathrm{mg}, \mathrm{mp} 240-241^{\circ}$ from EtOH, Lit. ${ }^{14} 240-241^{\circ}$ from $\left.\mathrm{CHCl}_{3}\right)$ and betulinic acid $(15.0 \mathrm{mg})$. The ethanol extract $(22.00 \mathrm{~g})$, was chromatographed on silica gel $(450.0 \mathrm{~g})$, with hexane, EtOAc and EtOH as eluents, giving chrysophanic acid (3, $8.5 \mathrm{mg}, \mathrm{mp} 191-193^{\circ}$ from acetone), friedelin $(12.0 \mathrm{mg}), \beta$-friedelinol $(8.0 \mathrm{mg})$, madagascine $(4,12.0$ $\mathrm{mg}, \mathrm{mp} 149.5-152.0^{\circ}$ from acetone, Lit. $^{7} \mathrm{mp} 154-156^{\circ}$ ) 1,7-dihydroxyxantone $(\mathbf{1}, 12.0 \mathrm{mg})$ betulinic acid $(15.0$ $\mathrm{mg}$ ) and 1,5-dihydroxy-8-methoxyxantone $(\mathbf{2}, 8.0 \mathrm{mg}, \mathrm{mp}$ 228,0-229, $8^{\circ}$ from EtOH, $\mathrm{Lit}^{14} \mathrm{mp} 230-231^{\circ}$ from $\mathrm{CHCl}_{3}$ ).

Extraction and isolation of the constituents from fruits: The fruits of Vismia parviflora, were dried and ground to a powder $(56.80 \mathrm{~g})$, which was throughly extracted with EtOH. Removal of solvent gave a residue $(16.94 \mathrm{~g})$, which was chromatographed on silica gel (370.00 g) using hexane, EtOAc and EtOH as eluents. Several frs. were collected and sepd. into five groups ( $\left.\mathrm{B}_{1}-\mathrm{B}_{5}\right)$ by TLC. $\mathrm{B}_{2}(0.56$ g) was washed with $\mathrm{Me}_{2} \mathrm{CO}$ and the insoluble portion (32.2 $\mathrm{mg})$ recrystalized in EtOH giving vismiaquinone $\mathrm{A}(\mathbf{6}, 18.0$ $\mathrm{mg}, \mathrm{mp}$ 197.3-198. $7^{\circ}$ from acetone, $\mathrm{Lit}^{8} \mathrm{mp} 202-204^{\circ}$ from petrol/EtOAc 19:1). The remaining soluble portion (0.40 $\mathrm{g}$ ), was purified by prep. TLC (layer thickness $1.0 \mathrm{~mm}$ ), giving vismiaquinone $\mathrm{C}\left(\mathbf{5}, 12.0 \mathrm{mg}, \mathrm{mp} 212,4-214,6^{\circ}\right.$ from acetone, Lit. ${ }^{10} 215-217^{\circ}$ from acetone), and madagascine $(4,13.5 \mathrm{mg}) . \mathrm{B}_{3}(0.21 \mathrm{~g})$, was recrystalized in $\mathrm{EtOH}$ giving chrysophanic acid $(3,17.0 \mathrm{mg})$. $\mathrm{B}_{4}(0.23 \mathrm{~g})$ was rechromatographed on sephadex LH 20 (sigma) with $\mathrm{MeOH}$ as eluent, yielding quercetin $(23.0 \mathrm{mg})$ and 3,4-dihydroxy- benzoic acid (13.0 mg). B 5 (4.30 g) was washed exhaustively with EtOH, giving shikimic acid (0.52 g).

\section{Acknowledgments}

Financial support from the Conselho Nacional de Desenvolvimento Científico e Tecnológico (CNPq), and Coordenação de Aperfeiçoamento Pessoal de Ensino Superior (CAPES) is gratefully acknowledged. The authors also thank anonymous referee for constructive suggestions.

\section{References}

1. Engler, A. Syllabus D. Pflanzenfamilien 12th; Ed.; G. Borntrager; Berlin, 1964, 2, p 444

2. Delle Monache, F. Rev. Latinoamer. Quim. 1985, 16, 5.

3. Index Kewensis and supplements 1-14, 1895, 1212.

4. Andrade, M.C.R.; Gotlieb, O.R. Biochem. Syst. 1973, 1,111 .

5. Delle Monache, G.; Gonzalez, J.G.; Delle Monache, F.; Marini Bettolo, G.B. Phytochemistry 1980, 19, 2025.

6. Gonzalez, J.G.; Delle Monache, F.; Delle Monache G.; Marini Bettolo, G.B. Planta Med. 1980, 40, 347.

7. Camele, G.; Delle Monache, F.; Delle Monache, G.; Marini Bettolo G.B.; De Lima, R.A. Phytochemistry 1982, 21, 417.

8. De Lourdes M.S.G.; Mors, W.B. Phytochemistry 1981, 20, 1947.

9. Miraglia, M.C.M.; Mesquita, A.A.L.; Verejão, M.C.J.; Gottlieb O.R.; Gottlieb, H.E. Phytochemistry 1981, 20, 2041.

10. Nagem, T.J.; Faria, T. De J. T. Phytochemistry 1990, 29,3362 .

11. Hostettmann, K.; Hostettmann, M. In Methods in Plant Biochemistry; Harborne, J.B., Ed. Academic Press, London, 1989, 1, 493.

12. Bowie, J.H.; White, P.Y. J. Chem. Soc. B 1969, 89.

13. Hill, R.A.; Krebs, H.C.; Verpoorte, R.; Wijnsma, R. In Progress in the Chemistry of Organic Natural Products; Ed. Springer-Verlag, New York, 1986, 49, p 84.

14. Delle Monache, F.; Mac-Quhae, M.M.; Delle Monache, G.; Marini Bettolo,G.B.; De Lima, R.A. Phytochemistry 1983, 22, 227.

15. Fujita, T.; Da-You, L.; Ueda, S.; Takeda, Y. Phytochemistry 1992, 31, 3997.

16. Arnone, A.; Nasini, G.; Vajna, O.P. Phytochemistry 1991, 30, 2729. 